\title{
Onsager's Conjecture on the Energy Conservation for Solutions of Euler's Equation
}

\author{
Peter Constantin ${ }^{1}$, Weinan $\mathbf{E}^{2}$, Edriss S. Titi ${ }^{3}$ \\ 1 Department of Mathematics, University of Chicago, Chicago, IL 60637, USA \\ 2 School of Mathematics, Institute for Advanced Study, Princeton, NJ 08540, USA \\ 3 Department of Mathematics and Mechanical and Aerospace Engineering, University of \\ California, Irvine, CA 92717, USA
}

Received: 17 November 1993

\begin{abstract}
We give a simple proof of a result conjectured by Onsager [1] on energy conservation for weak solutions of Euler's equation.
\end{abstract}

In this note, we give a simple proof of a result conjectured by Onsager [1] on the energy conservation for weak solutions of the 3D incompressible Euler's equation. To avoid questions regarding boundaries, we will assume periodic boundary conditions with period box $D=[0,1]^{3}$. We will use the summation convention and frequently suppress the independent variable $t$ for notational convenience. We use $B_{p}^{\alpha, q}$ to denote the Besov spaces.

Theorem. Let $u=\left(u_{1}, u_{2}, u_{3}\right) \in L^{3}\left([0, T], B_{3}^{\alpha, \infty}(D)\right) \cap C\left([0, T], L^{2}(D)\right)$ be a weak solution of the $3 D$ incompressible Euler's equation, i.e.

$$
\begin{gathered}
-\int_{0}^{T} \int_{D} u_{j}(x, t) \partial_{t} \psi_{j}(x, t) d^{3} x d t-\int_{D} u_{j}(x, 0) \psi_{j}(x, 0) d^{3} x \\
-\int_{0}^{T} \int_{D} \partial_{i} \psi_{j}(x, t) u_{i}(x, t) u_{j}(x, t) d^{3} x d t-\int_{0}^{T} \int_{D} \partial_{l} \psi_{i}(x, t) p(x, t) d^{3} x d t=0
\end{gathered}
$$

for every test function $\psi=\left(\psi_{1}, \psi_{2}, \psi_{3}\right) \in C^{\infty}\left(D \times R^{1}\right)$ with compact support. If $\alpha>\frac{1}{3}$, then we have

$$
\int_{R^{3}}|u(x, t)|^{2} d^{3} x=\int_{R^{3}}|u(x, 0)|^{2} d^{3} x, \text { for } t \in[0, T) .
$$

This is basically the content of Onsager's conjecture, except Onsager stated his conjecture in Hölder spaces rather than Besov spaces. Obviously the above theorem implies similar results in Hölder spaces. We state the results in the Besov spaces for two reasons: The first is that it gives the sharp result. The second is that the 
Besov norms are naturally related to the generalized structure functions. Onsager further conjectured that (2) may cease to be true for $\alpha \leqq \frac{1}{3}$. The significance of Onsager's conjecture can be appreciated in the context of Kolmogorov's theory of turbulence. This was also the motivation of Onsager. Kolmogorov predicts that in a fully developed turbulent flow, the energy spectrum $E(\cdot)$ in the inertial range is given by a power law:

$$
E(k)=C_{0} \bar{\varepsilon}^{\frac{2}{3}} k^{-5 / 3},
$$

where $\bar{\varepsilon}$ is the ensemble average of the energy dissipation rate $\bar{\varepsilon}=v\left\langle|\nabla u|^{2}\right\rangle$. One of the two key assumptions Kolmogorov made in order to obtain (3) is that $\bar{\varepsilon}$ is a positive finite number, independent of viscosity. Stated in physical space, (3) becomes

$$
\left\langle|u(x+r)-u(x)|^{2}\right\rangle^{\frac{1}{2}} \propto C_{0}|r|^{\frac{1}{3}}
$$

which means that $u$ has Hölder exponent $\frac{1}{3}$ in a statistically averaged sense.

The difference between (3-4) and Onsager's statement is that (3-4) is a statement about statistically averaged quantities, whereas Onsager's is path-wise.

In a series of two papers [2] Eyink has proved energy conservation (2) for the case when the Besov space is replaced by Hölder space $C^{\alpha}$ with exponent $\alpha>\frac{1}{2}$, and the case when $\alpha>\frac{1}{3}$ but with the Hölder norm replaced by a stronger norm

$$
\|u\|_{C_{*}^{\alpha}}=\Sigma_{k}|k|^{\alpha}\left|\hat{u}_{k}\right|, \hat{u}_{k}=\int_{D} u(x) e^{i k \cdot x} d^{3} x .
$$

The purpose of the present note is to give a simple proof for the sharp result in Besov spaces. The Besov space formulation corresponds to the following physical interpretation of the result: if

$$
\left\langle|u(x+r)-u(x)|^{3}\right\rangle^{\frac{1}{3}} \leqq C|r|^{\alpha}
$$

for $\alpha>\frac{1}{3}$ then $\bar{\varepsilon}=0$.

Proof: For the sake of simplicity, we will proceed as if the solution is differentiable in time. The extra arguments needed to mollify in time are straightforward. Let $\varphi \in$ $C_{0}^{\infty}\left(R^{3}\right)$ be a standard mollifier supported in $B(0,1), \varphi^{\varepsilon}(x)=\frac{1}{\varepsilon^{3}} \varphi\left(\frac{x}{\varepsilon}\right), v^{\varepsilon}=v * \varphi^{\varepsilon}$ for $v \in \mathcal{D}^{\prime}\left(R^{3}\right)$.

We have the following facts about functions in $B_{3}^{\alpha, \infty}$ :

$$
\begin{array}{r}
\left\|u(\cdot+y)-\left.u(\cdot)\right|_{L^{3}} \leqq C|y|^{\alpha}\right\| u \|_{B_{3}^{\alpha, \infty}}, \\
\left\|u-u^{\varepsilon}\right\|_{L^{3}} \leqq\left. C \varepsilon^{\alpha}\|u\|\right|_{B_{3}^{\alpha, \infty}}, \\
\left\|\nabla u^{\varepsilon}\right\|_{L^{3}} \leqq C \varepsilon^{\alpha-1}\|u\|_{B_{3}^{\alpha, \infty}} .
\end{array}
$$

Define

$$
r_{\varepsilon}(u, u)(x)=\int \varphi^{\varepsilon}(y)\left(\delta_{y} u(x) \otimes \delta_{y} u(x)\right) d^{3} y
$$


where

$$
\delta_{y} u(x)=u(x-y)-u(x) .
$$

Then it is easy to check that

$$
(u \otimes u)^{\varepsilon}=u^{\varepsilon} \otimes u^{\varepsilon}+r_{\varepsilon}(u, u)-\left(u-u^{\varepsilon}\right) \otimes\left(u-u^{\varepsilon}\right) .
$$

This is the main identity of this paper and holds pointwise.

Under the assumption of differentiability in time, we get from (1),

$$
\frac{1}{2} \frac{d}{d t} \int_{D}\left|u^{\varepsilon}(x, t)\right|^{2} d^{3} x=\int_{D} \operatorname{Tr}\left((u \otimes u)^{\varepsilon}\left(\nabla u^{\varepsilon}\right)\right) d^{3} x .
$$

Hence

$$
\int_{D}\left|u^{\varepsilon}(x, t)\right|^{2} d^{3} x-\int_{D}\left|u^{\varepsilon}(x, 0)\right|^{2} d^{3} x=2 \int_{0}^{t} d \tau \int_{D} \operatorname{Tr}\left((u \otimes u)^{\varepsilon}\left(\nabla u^{\varepsilon}\right)\right) d^{3} x .
$$

It can be easily checked that this last equality also holds when the differentiability assumption is replaced by $u^{\varepsilon} \in C\left([0, T] ; L^{2}(D)\right)$. Using (6), we get

$$
\left.\| r_{\varepsilon}(u, u)\right)\left\|_{L^{3 / 2}} \leqq C \varepsilon^{2 \alpha}\right\| u \|_{B_{3}^{\alpha, \infty}}^{2} .
$$

Since $\int_{D} \operatorname{Tr}\left(\left(u^{\varepsilon} \otimes u^{\varepsilon}\right) \nabla u^{\varepsilon}\right) d^{3} x=0$, we have from $(10)$,

$$
\begin{aligned}
& \left.\left|\int_{D}\right| u^{\varepsilon}(x, t)\right|^{2} d^{3} x-\int_{D}\left|u^{\varepsilon}(x, 0)\right|^{2} d^{3} x \mid \\
& \leqq C \int_{0}^{t} d \tau\left\{\left\|r_{\varepsilon}\right\|_{L^{3 / 2}}^{2 / 3}\left\|\nabla u^{\varepsilon}\right\|_{L^{3}}^{1 / 3}+\left\|u-u^{\varepsilon}\right\|_{L^{3}}^{2 / 3}\left\|\nabla u^{\varepsilon}\right\|_{L^{3}}^{1 / 3}\right\} \\
& \leqq C \varepsilon^{3 \alpha-1} \int_{0}^{t}\|u(\cdot, \tau)\|_{B_{3}^{\alpha, \infty}}^{3} d \tau \rightarrow 0
\end{aligned}
$$

as $\varepsilon \rightarrow 0$.

Acknowledgement. The work of Constantin was support by the DOE and NSF. The work of E was supported by the NSF, NEC and the Sloan Foundation. E also wants to thank the Department of Mathematics of The University of Chicago, where this work was completed, for its hospitality. The work of Titi was supported by NSF.

\section{References}

1. Onsager, L.: Statistical Hydrodynamics. Nuovo Cimento (Supplemento), 6, 279 (1949)

2. Eyink, G.: Energy dissipation without viscosity in ideal hydrodynamics, I and II. Preprint, 1992 
